\title{
INTEGRATED IDENTITY AND ACCESS MANAGEMENT SYSTEM FOR TERTIARY INSTITUTIONS IN DEVELOPING COUNTRIES
}

\author{
B. U. Stephen ${ }^{1,}{ }^{*}$, G. A. Chukwudebe ${ }^{2}$ and C. P. Ezenkwu ${ }^{3}$

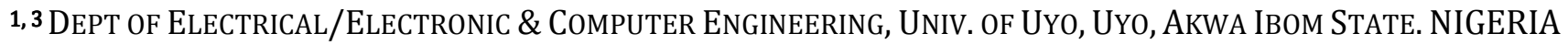

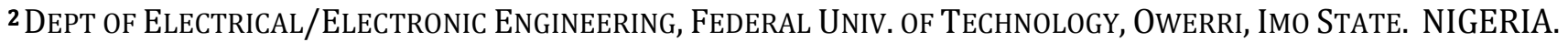 \\ E-mail addresses: ${ }^{1}$ blssstephen@yahoo.com, ${ }^{2}$ gloriachukwudebe@yahoo.com, ${ }^{3}$ edusco04@gmail.com
}

\begin{abstract}
Issuance of disparate identifiers to students, manual method of student identity management and access control and the unavailability of actionable information on pattern of students' use of schools' services describe identity and access management in tertiary institutions in developing countries. An Integrated Identity and Access Management System for tertiary institutions is developed in this work. This Tertiary Identity and Access Management System (T-IAMS) is a fingerprint biometric database that centrally manages students' identity, course registration, library and medical services information. It shortens student provisioning and de-provisioning processes, provides medical service data mining functionality and offers examination screening module which checks students into examination centres by cross-referencing database enrolment with course registration status. The system was developed following exploratory type of evolutionary software development model. MySql was used in building the database and Visual Basic for developing the graphic user interface of the student record module. The work also reports the results of testing done by 20 testers, on a 5-point scale. Analysis of the test results shows the system's average acceptability percentage of 100 in ability to prevent duplicate enrolments, 79.165 in effectiveness in student clearance while the format and usefulness of reports generated on the medical service module recorded 73.125 as average acceptability percentage. Also, effectiveness in examination screening averaged 66.785 in performance acceptability. It has been shown that managing student identity centrally eases student provisioning and de-provisioning processes, and tightens access control to school facilities and programmes.
\end{abstract}

Keywords: Biometrics, Identity and Access Management, Identifiers and Enrolment.

\section{INTRODUCTION}

The concept of identity and access management (IAM) has experienced a degree of maturity in higher education [1], but some problems solvable by the same concept persist. The underlying processes of identity and access management have been in use for many generations in an offline environment. For instance, Joao de Barros, a Spanish explorer and writer, wrote that early Chinese merchants used fingerprints to settle business transactions [2]. Babylonian business transactions were also recorded in clay tablets that included fingerprints [3]. Passports, driver's licenses, and employee ID cards are all components of what might be referred to as identity management systems - i.e., they are credentials issued by an entity for the purpose of identifying individuals, and they are used by such individuals to validate their identity in order to enter into a transaction with a third party. In Nigeria, National Identity Management Commission (NIMC), has rolled out National Identity Management System (NIMS) to integrate several government databases including those for driving licenses, voter registration, health insurance, taxes and pensions[4].In Nigerian banking industry, a centralized biometric identification system tagged Bank Verification Number (BVN) has been instituted. This number gives a unique identity that can be verified across the Nigerian Banking Industry [5].

In the Nigerian educational system, there has been relative extensive use of identity management 
involving the use of biometrics. For some time now, biometric registration for Unified Tertiary Matriculations Examination (UTME) has been in implementation. Biometric identification and authentication has been in extensive use at Covenant University, Ota, a Christian mission school in Nigeria. According to Pastor Michael Ogbolu, Director of Systems and Information Services, finger scanning biometrics, piloted in March, 2005, are currently in use for the weekly chapel attendance and for special meetings and in halls of residence [6].

However, in some institutions, student registration number, which is the only unique student academic data in use, does not reveal a student's department/course of study at a glance. This makes manual sorting and arrangement of students' record inefficient. Also, it is difficult for school management to capture important information such as the pattern of usage of the various school resources like the library, health centre, financial commitment etc. Adding to that is the isolated management of student identity which makes process of students' final year clearance (i.e. student de-provisioning) herculean. Along with increase in the number of students in tertiary institutions, has come the difficulty in controlling access to school facilities and programmes. The proliferation of services /resources offered by tertiary institutions of learning has resulted in the issuance of multiple identifiers to students. This is inefficient and uneconomical, thus raising the need for a centralized management of student identity. Increased familiarity with biometrics, from its widening use, motivated the use of biometrics (fingerprint) in solving the problems identified in this work.

\section{REVIEW OF IDENTITY AND ACCESS MANAGEMENT SYSTEMS}

There are three ongoing implementations similar to this project reviewed in this section: India's UID project, Covenant University Database Management System and Examination Screening and Attendance Monitoring Model in Yaba College of Technology.

Unique Identity Authority of India (UIDAI) has rolled out an identity management project to uniquely identify Indian residents - robust enough to eliminate duplicates and fake identities[7].Zelazny[7] also writes that enrollee's data is submitted to the Central Identity Data Repository (CIDR)of the UIDAI project. The CIDR then runs a de-duplication check to ensure that the resident is not already enrolled. The fact that the UID system is both de-duplicated and universal discourages residents from giving incorrect data at the time of enrolment. India's UID project uses fingerprint and iris as biometric traits[7].

The Covenant University Database Management System, a biometric database used in Covenant University, Ogun State, Nigeria, stores university basic data, student records (i.e. bio data, school fee records of students) and courses (courses offered by programmes, course listing, course allocation, course assessment plan etc.). There are modules in the Covenant University Database System that supply above listed services[6]. All modules/interfaces connect to the same database backend. There might be a connection between some health complains and study environments which a health service module can confirm or dispute. All interfaces in the Covenant University Database Management System connect to the same database backend developed with MySql. Students update their data by themselves[6].

As captured by Rufai et al [8], the Biometric Examination Screening and Attendance Monitoring System (BESAMS), currently implemented in Yaba College of Technology, Lagos, Nigeria, comes in two parts: enrolment and verification. During enrolment, a student registers for courses by filling an online course registration form and his biometric data is captured and stored. The verification takes place before the commencement of an examination. It involves an examination candidate appending his finger on a fingerprint scanning device for data capture. Rufai et al [8] adds that with the capturing of biometric data in BESAMS, studentship, course registration and fee payment status are then checked. The student is granted access if all these are confirmed correct and up to date. This approach means there might be a setback if biometric detection devices are for some reason not available and if the quality of a student's fingerprint is compromised.

\section{METHODOLOGY}

Tertiary Identity and Access Management System (TIAMS) developed in this work generates new registrations numbers for students, connects the individual information systems, manages student records centrally and generates actionable data for school management especially from student health history. The system also ensures that only legitimate students are allowed access to the university facilities and programmes at any given time. It also offers administrators irrefutable record of the time and date 
of each person's entry into examination/lecture halls, library etc. With central management of student records, the system improves the processes and workflows for student provisioning and deprovisioning as well as reducing the number of identities (IDs) issued to just one.

T-IAMS as depicted in Figure 1 involves a relational database as backend and two frontends: a windowbased application and a web-based application.

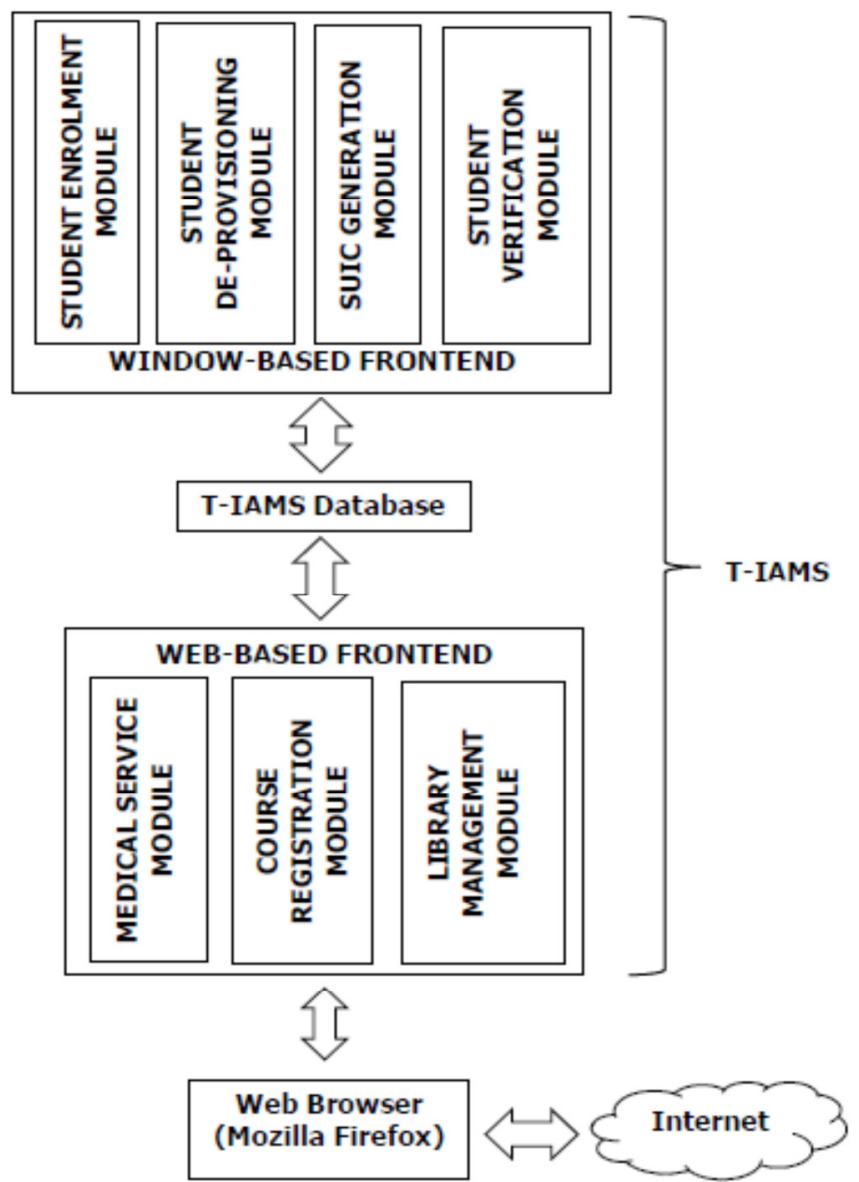

Figure 1: Block diagram showing Software Architecture of T-IAMS.

As shown also in figure 1, T-IAMS is limited to medical service, course registration and library information systems. The student examination screening module checks only for student's enrolment status and course registration status.

The Relational Database developed with MySql, stores academic, personal and bio (fingerprint) data from and supplies same to the two frontends. The fingerprint data collected are converted into alphanumeric strings of numbers that make meaning only to the fingerprint biometric algorithm in this system. The fingerprint data is stored in this form, making it difficult to recreate a fingerprint from the mathematical representation. The database also gives statistical information on the data stored. This is especially important for report generation, a key objective of this work. The database interacts with the internet through the Apache web server.

\subsection{Development of T-IAMS}

Building of the database in T-IAMS began with requirement analysis, which involved drafting of sample input forms and sample queries and reports. Next, data needed like personal and bio data needed were identified and organized into tables. Relationships were then created among the tables. Finally, to reduce data redundancy, the design was normalized up to the third normal form (3NF).

Visual Basic was used for the graphic user interface (GUI) of the window-based frontend while Dreamweaver was preferred for the GUI of the webbased frontend. The window-based frontend has interfaces for student enrolment, examination screening and student clearance/de-provisioning while the web-based frontend has modules for student course registration, library and medical service management.

A personal computer with webcam and fingerprint scanner forms the hardware setup for administration of T-IAMS.

\subsection{Implementation of Window-based Frontend}

\section{A. Enrolment Module}

The Use case diagram in figure 2 shows various functionalities of this module while figure 3 shows the student enrolment process. The enrolment process begins with capture of student personal and bio data after staff login. Data de-duplication is performed next. The system only proceeds to generate the Student Unique Identification Code (SUIC) and electronic copy of student ID if the new fingerprint template is not already in the database. SUIC is of the format: year of admission/Faculty/Department/4digit unique number. Faculty and department information are abbreviated. The average enrolment time is 7 minutes.

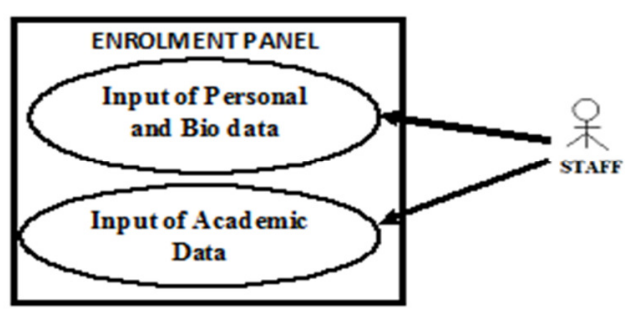

Figure. 2: Use case Diagram Showing the Functionalities in the Student Enrolment Panel. 


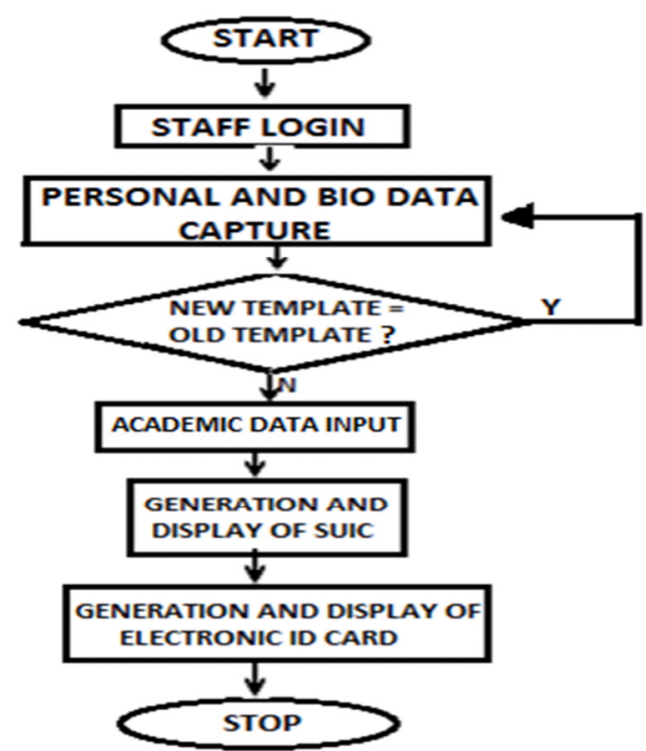

Figure.3: Flowchart showing the student enrolment process in T-IAMS.

\section{B. Examination Screening and Attendance List Compilation Module}

Clicking on Student Verification icon opens this module. It provides fields for input of year, department, course and capture of fingerprint. It compiles a list of students screened. For this screening, the claimant's fingerprint is compared with templates in the database to ascertain enrolment and course registration status. SUIC can be used in place of fingerprint if a fingerprint scanner is unavailable. This module authorizes entry if the claimant has enrolled in the database and has registered for the course of interest. However, this module is used in combination with manual matching of student features and data on the ID card. This use of two-factor authentication discourages impersonation attempts, resulting in reduced false acceptance rate since false acceptance can only occur when unauthorized access (impersonation) is attempted. Figure 4 illustrates the various functionalities of the examination screening module.

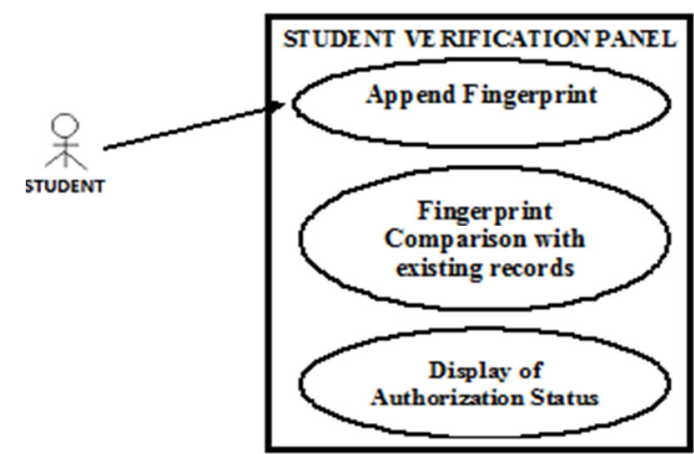

Figure 4: Use case diagram showing the functionalities of Student Verification/Examination Screening Panel.

\section{Student Clearance/deprovisioning}

T-IAMS provides the functionality of compiling the status of students across the various information systems. This panel checks this and compiles a list of cleared students. A student is cleared if he is cleared in the individual service providers/interfaces - no unreturned library materials and no outstanding courses. Figure 5 shows the use cases of the student de-provisioning panel.

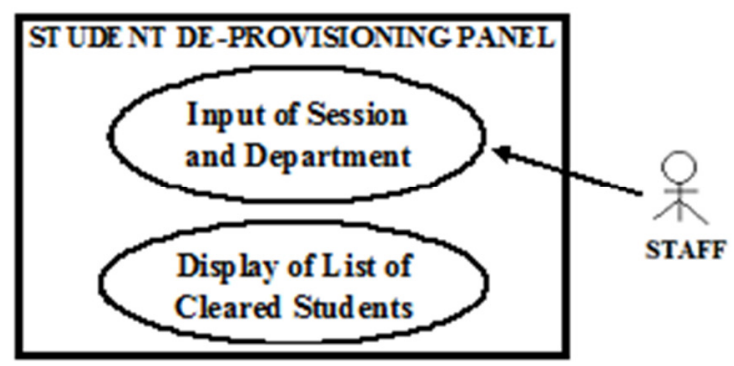

Figure 5: Usecase diagram showing the functionalities of Student Clearance/De-provisioning Panel.

\subsection{Implementation of Web-based Frontend}

\section{A. Student Course Registration}

The student course registration process, begins with the student logging on with his SUIC and password. He then selects the session, semester and course. Successful registration for a course is only possible if the selected course has not been previously registered for in the semester under review and if the maximum credit unit for a single semester is not exceeded. This is the only interface in T-IAMS directly accessible to students.

\section{B. Library Management Module}

The use of Library Management Module is intuitive and simple. A student's SUIC is used to call up his account where his various transactions with the library are viewed and if necessary updated.

\section{Health Management Information System Module}

The incorporation of T-IAMS will give the management of student identity an improved look in terms of provisioning and de-provisioning. On successful enrolment into T-IAMS, accounts are automatically created for the student in the Library and Health Centre information systems. This makes separate registration into these information systems unnecessary. Only a single ID card will be issued to a student on registration. SUIC is an alternative to ID card. Figure 6 shows the use cases of the health information system module. 


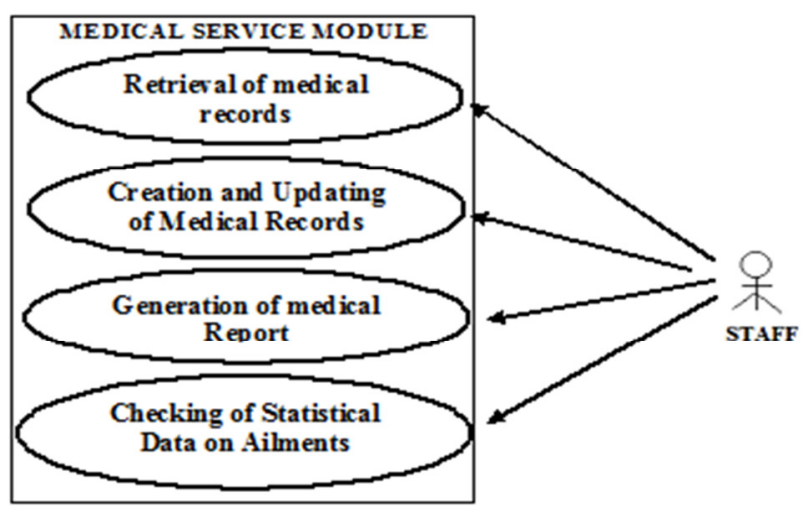

Figure 6: Usecase Diagram Depicting the

Functionalities in the Medical Service Module.

A student on completion of studies is de-provisioned centrally. A list of cleared students in a department for a particular session is automatically compiled and printed out on the Student Clearance Panel in T-IAMS. Only students with no outstanding course and no unreturned library material can have their names on this list.

\section{TESTING AND RESULTS}

The system testing was performed by 20 independent testers and their responses were collected using a questionnaire with a 5-point Likert scale: very dissatisfied, dissatisfied, neutral, satisfied and very satisfied. The Likert items or test cases used for the testing were the system modules. For each module, respondent or tester selected one of the radio buttons labeled very dissatisfied, dissatisfied, neutral, satisfied and very satisfied. For analysis these were assigned numeric values as follows; very dissatisfied $=1$, dissatisfied $=2$, neutral $=3$, satisfied $=4$ and very satisfied $=5$.

\subsection{Test Results}

The detailed responses from the respondents are presented in the Appendix A. Bar charts showing the degree of acceptability of each module of the system were obtained using the MatLab code in Appendix B. The bar charts are presented in figure7.

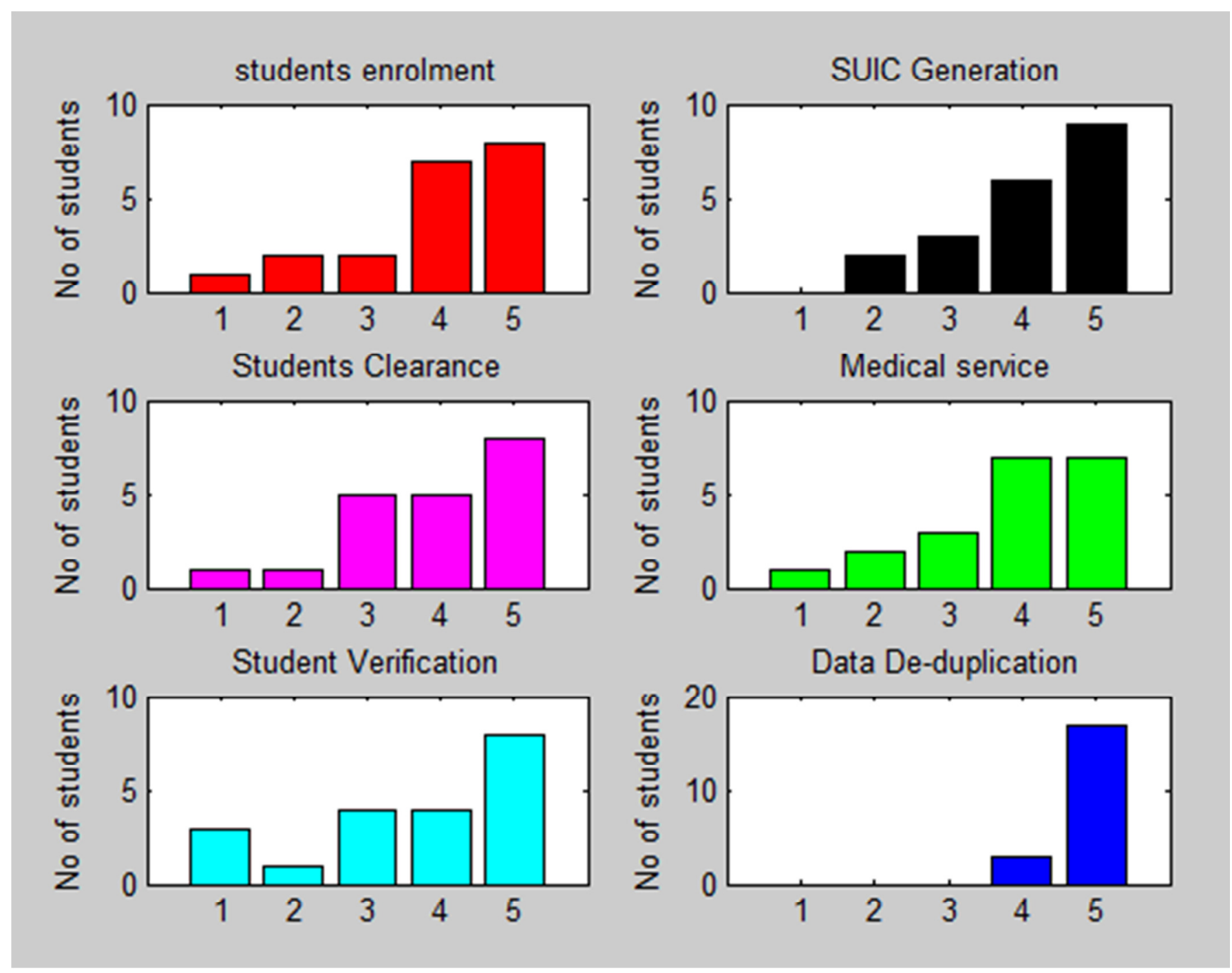

Figure7: Bar chart showing the degree of acceptability of each module. 


\subsection{Analysis of Results}

The percentage acceptability of each module considering only testers who were not neutral is calculated by:

$$
P_{a 1}=\frac{T 2}{T T-T N} \times 100 \%
$$

The percentage unacceptability of each module considering only testers who were not neutral is calculated by:

$$
P_{u 1}=\frac{L 2}{T T-T N} X 100 \%
$$

The percentage acceptability of each module considering all testers is calculated by:

$$
P_{a 2}=\frac{T 2}{T T} X 100 \%
$$

The percentage unacceptability of each module considering all testers is calculated by:

$$
P_{u 2}=\frac{L 2}{T T} \times 100 \%
$$

Where, T2 is total number of the two topmost scores i.e. satisfied (4) and very satisfied (5).

L2 is total number of the two lowest scores i.e. dissatisfied (2) and very dissatisfied (1),

TT is the total number of Testers,

TN is the total number of Neutral respondents,

$P_{a i}$ is percentage acceptability

$P_{u i}$ is percentage unacceptability

$\forall i \in\{1,2\}$ such tha $i=1$ implies that neutral respondents are neglected, and $i=2$ means that neutral respondents are considered.

Average percentage acceptability of the entire system is given by:

$$
P_{a s}=\sum_{i=1}^{N} P_{a v}(i){ }_{N}=\frac{462.575}{6}=77.096 \%
$$

Average percentage unacceptability of the entire system is given by:

$$
P_{u S}=\frac{\sum_{i=1}^{N} P_{u v}(i)}{N}=\frac{97.420}{6}=16.237 \%
$$

Noticeably, $\mathrm{P}_{\mathrm{as}}+\mathrm{P}_{\mathrm{us}}<100$, because the average percentage neutrality was neglected since it gives no information about the system acceptability.

Hence, the average percentage neutrality of the System,

$$
P_{n S}=100-\left(P_{a s}+P_{u S}\right)=66.667 \%
$$

\section{DISCUSSION OF RESULTS \\ 5.1 Student Enrolment}

This module enables the capturing of sufficient fields of student data as well as the turnaround time of 8 minutes per enrolment gave this module an average percentage acceptability of 87.055. More information integrity is offered by staff of the university handling the enrolment and update processes as opposed to the scenario in some cases investigated.

\subsection{Medical Service Module}

This module or its equivalent is not available in some of the cases investigated. This module captures nearly as much information as manual hospital records and simplifies access to school health care, a combination of which can effectively replace the manual system in use. The module also avails school management with

\begin{tabular}{|c|c|c|c|c|c|c|c|}
\hline $\mathrm{S} / \mathrm{N}$ & TEST CASES & $\begin{array}{l}\mathrm{Pa}_{1} \\
(\%)\end{array}$ & $\begin{array}{l}\text { Pu1 } \\
\text { (\%) }\end{array}$ & $\begin{array}{l}\mathrm{Pa} 2 \\
(\%)\end{array}$ & $\begin{array}{l}\mathrm{Pu} 2 \\
(\%)\end{array}$ & $\begin{array}{l}\text { Av. } \mathrm{Pa}(\%) \\
\quad P a v=\frac{P a 1+P a 2}{2} \\
\text { (for each module) }\end{array}$ & $\begin{array}{l}\text { Av. } \mathrm{Pu}(\%) \\
\quad P u v=\frac{P u 1+P u 2}{2} \\
\text { (for each module) }\end{array}$ \\
\hline 1. & Student Enrolment & 94.11 & 5.88 & 80 & 5 & 87.055 & 5.44 \\
\hline 2. & Medical Service Module & 81.25 & 18.75 & 65 & 15 & 73.125 & 16.875 \\
\hline 3. & $\begin{array}{l}\text { Student Clearance and } \\
\text { Component } \\
\text { Interconnectivity }\end{array}$ & 83.33 & 16.67 & 75 & 15 & 79.165 & 15.835 \\
\hline 4. & SUIC Generation & 57.89 & 42.11 & 55 & 40 & 56.445 & 41.055 \\
\hline 5. & Student Verification & 78.57 & 21.43 & 55 & 15 & 66.785 & 18.215 \\
\hline 6. & Data De-duplication & 100 & 0 & 100 & 0 & 100 & 0 \\
\hline
\end{tabular}
information regarding pattern of student use of the Medical Services, prevalent health challenges and the department whose students come up with these complaints.

Table 1: Table showing Average Acceptability and Average Unacceptability Percentage of the various Modules on T-IAMS. 


\subsection{Student Clearance and Component Interconnectivity} Creation of student records with the various service providers only on single registration shows good component interconnectivity. Student clearance (user de-provisioning) is just as easy. The average percentage acceptability of 79.165 , as shown in table 1 , supports the claim.

\subsection{SUIC Generation}

With SUIC, a student's faculty, department and year of admission can be identified at a glance.

\subsection{Student Verification}

The use of verification panel meets as many functions that are expected, ranging from tying authentication at examination/test venues to course registration, to the printing of list of authenticated students. It however pales in comparison with the biometric examination screening in some institutions studied, which screens students into examination venues by crossreferencing database enrolment, class attendance, fee payment and course registration status of candidates.

\subsection{Data De-duplication}

This would not be a successful IAM system if multiple enrolments were allowed. Fingerprint, as it does in India's UID project, proved a suitable biometric trait in data de-duplication in this work. This is reflected by the perfect average percentage acceptability of 100 , as shown in Table 1.

\section{CONCLUSION}

As demonstrated by test results, this project has showed that student identity can be managed centrally and this will afford school authorities the efficiency in controlling access to school programmes and facilities. Provisioning and de-provisioning processes have been eased by having one unified identity that covers all the information systems. Finally, having an electronic repository of student records makes it easier to implement statistical tools for data mining.

\section{RECOMMENDATIONS}

This application, as resourceful as it has proven, can be further improved upon. A more holistic examination screening can be achieved with crossreferencing student enrolment in the database with fee payment, course registration and class attendance status. The library management module can be made to generate important reports regarding student study patterns, preferred books (by extension, ignored books) and average length of study time.

\section{REFERENCES}

[1] Aegis Identity Software, Inc. "Trends in Identity \& Access Management Solutions in Higher Education Institutions", 2012.

[2] Roberts,

"Biometrics"http://www.ccip.govt.nz/newsroom/inf ormation-notes/2005/biometrics.pdf, Accessed on July 15, 2015.

[3] Unger, R. Life prints, Berkeley, CA: Crossing Press, 2007.

[4] "Nigeria Launches National Electronic ID Cards", $B B C$ News (August 28, 2014)..

[5] "13 Things bank customers must know about Bank Verification Number", Premium Times, (November 15, 2014)..

[6] "Centre for Systems and Information Services (CSIS) Overview"

www.covenantuniversity.edu.ng/content/download 44624/CSIS Brochure.pdf Accessed on July 14, 2015.

[7] "Developing Countries" Centre for Global Development (CGD) Policy Paper 008.

[8] Rufai, M. M., Adigun, J. O., \& Yekini, N. A."A Biometric Model for Examination Screening and Attendance Monitoring in Yaba College of Technology". World of Computer science and Information Technology Journal (WCSIT),2 (4), pp. 120-124, 2012.

[9] Bruhn, A., Gettes M., \& West, A."Identity and Access Management and Security in Higher Education", Educause Quarterly, No. 4, 2003.

[10] Camp, J. "Digital Identity". IEEE Technology and Society Magazine, Fall, pp.34-41, 2010.

[11]. UIDAI, "Ensuring Uniqueness: Collecting Iris Biometric for the Unique ID Mission",www.uidai.gov.in/UID_PDF/Working_Pape rs/UID and iris paper final.pdf, Accessed May 31, 2014.

[12]. Georgia State University, "Building an Identity Management Infrastructure for the e-University", NMI Integrated Testbed, 2014.

[13] Manivannan, N., Tigli, C., Noor, A., \& Memon, S. "Fingerprint Biometric for Identity management", International Journal of Industrial Engineering and Management (IJIEM), 2 (2), pp. 39-44, 2011.

[14]. Centre for Educational Performance and Information (CEPI), "MEIS/Single Record Student Database/Unique Identification Code (SRSD/UIC) Application/User's Guide", 2008. 
[15] Iloanusi, O. N. and Osuagwu C. C., "Biometric Recognition: Overview and Applications", Nigerian Journal of Technology, Vol. 27, No. 2, 2008, pp. 3645.
[16] Iloanusi, O. N. and Osuagwu C. C., "IMAGE Processing And Features Extraction of Fingerprint Images", Nigerian Journal of Technology, Vol. 29, No. 2, 2010, pp. 21-31.

\section{APPENDIX A}

\begin{tabular}{|c|c|c|c|c|c|c|c|}
\hline $\mathrm{S} / \mathrm{N}$ & TESTERS & $\begin{array}{l}\text { Student } \\
\text { Enrolment }\end{array}$ & $\begin{array}{l}\text { Medical } \\
\text { Service } \\
\text { Module }\end{array}$ & $\begin{array}{l}\text { Student Clearance } \\
\text { And } \\
\text { Component } \\
\text { Interconnectivity }\end{array}$ & $\begin{array}{l}\text { SUIC } \\
\text { Generation }\end{array}$ & $\begin{array}{l}\text { Student } \\
\text { Verification }\end{array}$ & $\begin{array}{l}\text { Data } \\
\text { De- } \\
\text { duplication }\end{array}$ \\
\hline 1 & TESTER 1 & 5 & 4 & 2 & 4 & 1 & 5 \\
\hline 2 & TESTER 2 & 3 & 4 & 5 & 1 & 4 & 5 \\
\hline 3 & TESTER 3 & 4 & 5 & 5 & 1 & 5 & 5 \\
\hline 4 & TESTER 4 & 3 & 5 & 4 & 3 & 4 & 5 \\
\hline 5 & TESTER 5 & 4 & 5 & 5 & 2 & 3 & 5 \\
\hline 6 & TESTER 6 & 5 & 3 & 5 & 4 & 3 & 5 \\
\hline 7 & TESTER 7 & 5 & 5 & 4 & 5 & 5 & 5 \\
\hline 8 & TESTER 8 & 5 & 4 & 5 & 4 & 3 & 5 \\
\hline 9 & TESTER 9 & 4 & 4 & 5 & 1 & 1 & 5 \\
\hline 10 & TESTER 10 & 5 & 3 & 1 & 5 & 4 & 5 \\
\hline 11 & TESTER 11 & 4 & 2 & 5 & 4 & 3 & 5 \\
\hline 12 & TESTER 12 & 4 & 5 & 4 & 4 & 3 & 5 \\
\hline 13 & TESTER 13 & 5 & 4 & 4 & 2 & 3 & 5 \\
\hline 14 & TESTER 14 & 3 & 5 & 2 & 4 & 5 & 5 \\
\hline 15 & TESTER 15 & 4 & 1 & 5 & 2 & 4 & 5 \\
\hline 16 & TESTER 16 & 5 & 5 & 5 & 1 & 5 & 5 \\
\hline 17 & TESTER 17 & 4 & 1 & 3 & 5 & 5 & 5 \\
\hline 18 & TESTER 18 & 2 & 3 & 5 & 5 & 2 & 5 \\
\hline 19 & TESTER 19 & 4 & 5 & 3 & 2 & 5 & 5 \\
\hline 20 & TESTER 20 & 5 & 3 & 4 & 5 & 5 & 5 \\
\hline
\end{tabular}

\section{APPENDIX B}

load ('data.csv') sz is the size(data); col = 1; Line_color = ['r','k','m','g','c','b'];

while $\mathrm{col}<=6$

$\mathrm{a}=$ find $($ data $(:, \mathrm{col})==1)$;

$\mathrm{b}=$ find $($ data $(:, \mathrm{Col})==2)$;

$\mathrm{c}=$ find $($ data $(:, \mathrm{col})==3)$;

$\mathrm{d}=$ find $($ data $(:, \mathrm{col})==4)$;

$\mathrm{e}=$ find $($ data $(:, \mathrm{col})==5)$;

subplot(3,2,col) bar([1 $\left.\begin{array}{llll}1 & 2 & 4 & 5\end{array}\right]$,[length(a) length(b) length(c) length(d) length(e)],Line_color(col)) ylabel ('No of students') if $\mathrm{col}==1$, title' student enrolment' elseif $\mathrm{col}==2$, title 'Medical service', elseif $\mathrm{col}==3$ title'Student Clear' elseif $\mathrm{col}==4$, title'SUIC Generation' elseif $\mathrm{col}==5$ title'Student Verification' else title 'Data De-

duplication' end col $=$ col +1 ; end 